\title{
Effects of the change in auto coupler parameters on in-train longitudinal forces during brake application
}

\author{
Saeed Mohammadi ${ }^{1}$ And Reza Serajian ${ }^{2, a}$ \\ 1 Mechanical Engineering Department, IUST University, Iran \\ 2 Mechanical Engineering Department, KNTU University, Iran
}

Received 17 April 2013, Accepted 1 September 2014

\begin{abstract}
For long freight trains, the pneumatic brake systems are still used. In these situations, the cars at the end of the train brake a few seconds later than those wagons at the front. This variable braking action along the train may cause large longitudinal forces and impacts. Beside brake system parameters, auto coupler characteristics parameters have high importance in longitudinal train dynamics. Therefore, simulations of effects of the change in auto coupler parameters on in-train longitudinal forces during brake application are very important both for safety and economic reasons since longer freight trains imply higher transport costs reductions. In the present paper, effects of the change in coupling parameters such as stiffness, damping, and clearance and train speed, on the longitudinal train dynamics were investigated during brake application. The results indicate dramatic changes in the way that cars interact dynamically with each other and the magnitudes of the in-train longitudinal compression and tensile forces along the train. The freight train considered is in current use by Iranian Railways.
\end{abstract}

Key words: In-train longitudinal forces / nonlinear dynamics / stiffness / damping / clearance / brake delay time

\section{Symbols and Notations}

\begin{tabular}{|ll|}
\hline$\varphi$ & Slope of the friction wedge \\
$\lambda$ & Constant, which depends on the car brake system \\
$\mu_{\mathrm{s}}$ & Static $c$ friction coefficient \\
$\eta_{\mathrm{dyn}}$ & Dynamic efficiency of the rigging ratio \\
$\mu_{\mathrm{k}}$ & Dynamic friction coefficient \\
$\beta$ & Friction curve gradient around the \\
& point where the friction polarity changes \\
\hline
\end{tabular}

\section{Introduction}

Longitudinal train dynamics, as an important problem in railway systems, commonly considered as the motions of the cars and locomotives of a moving train and the relevant in-train forces between the cars along the trains. Train motion includes the motion of the train as a whole and the relative motions between vehicles (wagons and locomotives) due to the looseness of the couplers between the vehicles as well as the elasticity of the vehicles' bodies and their coupling systems. Longitudinal train dynamics depend on a number of parameters such as operational parameters, load distribution of wagons, coupler's dynamic specifications, train forward velocity..., and train brake delay time between the cars. Hence, investigation on train longitudinal dynamics and specifying the optimal design parameters is complicated. However, in the last two decades, longitudinal train dynamics studies have been directed to understand and reduce longitudinal in-train forces and to enhance dynamic behavior of the trains. In 1984, Rao et al. studied the transient response and coupler (draw bar) forces of a train-consist subjected to different train handling conditions [1]. In 1989, measurements and simulations of in-train forces in the Queensland coal trains were reported by Duncan and Webb [2] and later by Jolly and Sismey similar investigations were carried out regarding trains of double existing length in New South Wales railways [3]. In 1984, Fukazawa calculated the coupler forces of trains made of two-axle freight cars during train emergency braking. It is concluded that at speeds lower than $30 \mathrm{~km} / \mathrm{h}$ emergency braking should not be applied because in low speeds, the difference between kinetic energies in wagons during braking (braking force is applied gradually among wagons) causes high-speed differential that leads harsh impacts between wagons [4].

\footnotetext{
${ }^{a}$ Corresponding author: reza.serajian@yahoo.com
} 


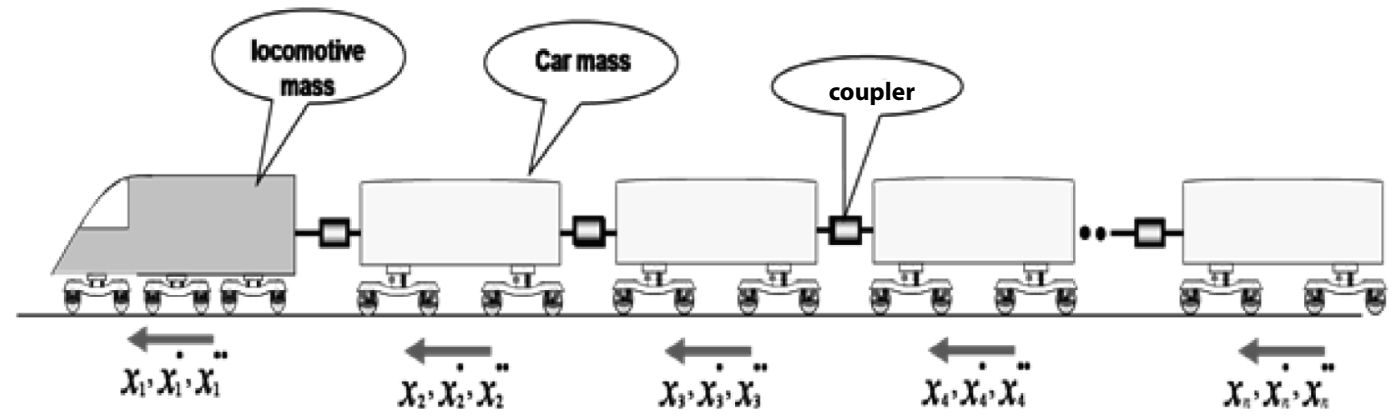

Fig. 1. Longitudinal forces in train-consist of $n$ vehicles each having a mass of $m$.

In 1993, El-Siabie reported that lateral and vertical components of forces resulting from coupler angles on horizontal and vertical curves are also quite large [5]. These components will adversely affect wagon stability with the possibility of train derailment at some points. In 1995, Zobory et al. [6] attempted to simulate longitudinal train dynamics, focusing on the track geometry. In 1996, Barbosa et al. in Brazil studied the longitudinal train dynamics in long freight trains [7]. Oyan in China studied the longitudinal dynamics of a typical subway train in 1998 [8]. In this study, simplified train dynamics for the trains in Taipei Metro to avoid possible dangerous conditions of coupler failure and to understand the deformation of the "mechanical fuse" in a train collision was studied. It is found that sudden failure of couplers would not happen for a train at speeds up to $5 \mathrm{~km} / \mathrm{h}$. Coupler force for a train running at a normal acceleration from the start or at an emergency stop is also investigated. In addition, it is found that damage is confined to the fuse structures and the fuse deformation is less than the maximum allowable deformation designed in the trains, under an impact speed of $25 \mathrm{~km} / \mathrm{h}$.

Verbitskiy et al. reported similar results for a monorail car in 2000 [9]. They looked at the relationship between lateral in-train coupler force components and wheel unloading. Mcclanachan et al. reported different modes of dynamic interaction in 1999 [10].

Ansari et al. studied the sensitivity analysis in longitudinal freight trains dynamics in 2008 [11]. In this research, effects of different operating parameters (such as stiffness and damping of automatic couplers, train forward speed, coupler clearance, and train acceleration and braking processes) on longitudinal train dynamics were investigated parametrically. The same investigators also investigated effects of load distribution patterns on the longitudinal freight train dynamics in 2009 [12]. In year 2010, Nasr and Mohammadi investigated effect of brake delay time on in-train longitudinal forces. In the same year, effect of power unit location on in-train longitudinal forces was investigated by the same authors.

In the present paper, using simulation technique, the effects of the change in coupling parameters on in-train longitudinal forces during brake application are investigated. The model is nonlinear. An extensive parametric study is carried out to find out how changes in the design and operational parameters. Such as changes in stiffness, damping, and clearance and train speed affect the longitudinal train dynamics. This study is performed on a freight train-consist of one locomotive and fifty-two wagons. All the input parameters are in full accordance with those currently used in Iranian railways.

\section{Train longitudinal dynamic modeling}

Figure 1 shows the physical model for determination of the longitudinal forces in a train. Here, a system of coordinate is attached to each of the cars (wagons and locomotive). In this system $x$ is displacement length in the direction of movement, $\dot{x}$ is velocity, and $\ddot{x}$ denotes acceleration of the individual vehicles. The longitudinal dynamic behavior of a train can be described by a system of differential equations. For setting up the relevant equations, it is usually assumed that there is no lateral or vertical movement of the cars. The required governing differential equations can be developed by considering the generalized $n$ mass dynamic system. During train application of brake, relative movements occur between individual masses.

On each wagon act a brake force $F_{\mathrm{B}}$, two coupler (buffer) forces $F_{\mathrm{WC}}$ and a resistance force $F_{\mathrm{R}}$. On each locomotive, one might add a traction force as well. The cars are connected with buffers equipped with springs for push-pull modes. Depending on the compression or tensile of the springs of the buffers a push or pull force results. Considering each car as a rigid mass, the train can be described as a $n$ point mass dynamic system. The equations of motion for individual masses are as follows:

For the leading vehicle (the locomotive) in-train:

$$
m_{1} \ddot{x}_{1}+f_{\mathrm{wc}}\left(\dot{x}_{1}, \dot{x}_{2}, x_{1}, x_{2}\right)=-f_{\mathrm{B}, 1}-f_{\mathrm{R}, 1}+f_{\mathrm{tr}}
$$

For the wagon $i$ in the train,

$$
\begin{aligned}
m_{i} \ddot{x}_{i}+f_{\mathrm{wc}} & \left(\dot{x}_{i}, \dot{x}_{i-1}, x_{i}, x_{i-1}\right) \\
& +f_{\mathrm{wc}}\left(\dot{x}_{i}, \dot{x}_{i+1}, x_{i}, x_{i+1}\right)=-f_{\mathrm{B}, i}-f_{\mathrm{R}, i}
\end{aligned}
$$

For the $\mathrm{n}$ th or the last wagon of the train:

$$
m_{n} \ddot{x}_{n}+f_{\mathrm{wc}}\left(\dot{x}_{n}, \dot{x}_{n-1}, x_{n}, x_{n-1}\right)=-f_{\mathrm{B}, n}-f_{\mathrm{R}, n}
$$

In the above equations, $f_{\mathrm{wc}}$ is a nonlinear function describing the full characteristics of the couplers. 


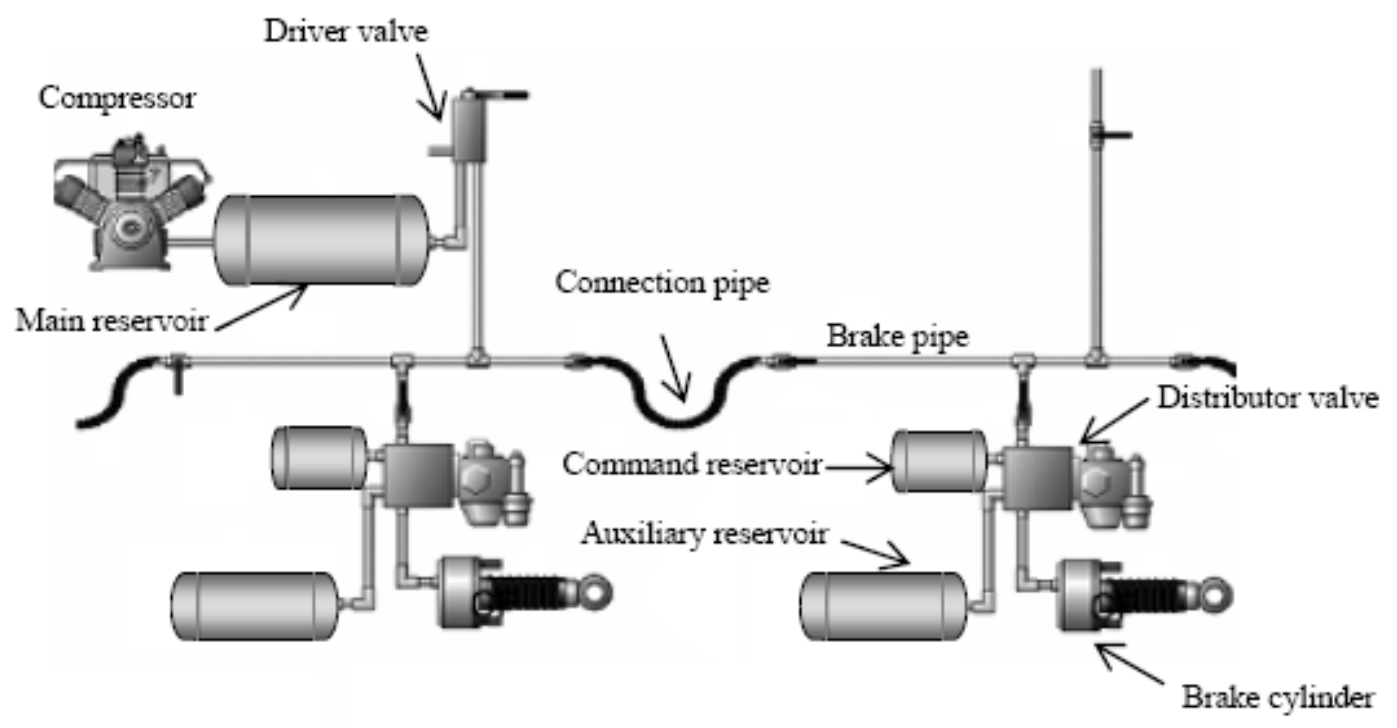

Fig. 2. Schematic of automatic air brake system.

Solution to the above set of equations is further complicated by the need to calculate the forcing inputs to the system, i.e., $F_{\mathrm{tr}}, F_{\mathrm{R}}$, and $F_{\mathrm{B}}$. Approaches to the nonlinear modeling of the wagon connections and of the forcing inputs are included and discussed below.

\section{Pneumatic brake modeling}

Wagons' braking system is a pneumatic one in which air pressure inside braking cylinders gives rise to a clamping force that is used to activate a disk or block brake against the rotating wheels [1]. In Figure 2, a simplified scheme of the pneumatic brake system is shown.

Variable pressure inside brake cylinder is used to modulate the braking effort. In trains equipped with the pneumatic brake, every vehicle is provided with a car distributor valve. This device modulates the pressure inside brake cylinder according to a law that is proportional to the difference between the pressure in the air brake pipe and the reference pressure stored in the command reservoir unit. More precisely, for safety reasons, the braking is activated by a reduction of the air brake pipe pressure of at least 0.15 bar with a pressure gradient of at least 100-200 mbars/s from a reference pressure of about five bars. The driver using a brake valve that can be roughly modeled by a programmable pressure regulator according to the desired maneuvers generates depression signal in the brake pipe. In order to optimize both braking and comfort performances, the distributor valve gives a highly non-linear/gain and works as a scheduled pressure regulator with variable dynamic behavior, according to the pressure and its gradient values in both brake pipe and cylinder.

In this air brake system, braking of the train starts by reducing the air pressure (introducing brake signal) in the train main air brake pipe. The brake control valve located in the locomotive cab applies this. By reducing the air pressure in the main pipe, the distributor valve of each car becomes activated which causes flow of the air from the car auxiliary air reservoir to the brake cylinder. The cylinder thrust produced is provided to the rigging system such as shown in Figure 3, then finally transferred to the brake shoes. Friction force induced between brake shoes and wheels causes braking effort.

Regarding train operation, it is a good practice that the brake force is applied simultaneously in all wagons along the train. However, in-trains having a length of a couple of hundred meters, the pressure wave (brake signal) in air brake pipe reaches the end of the train approximately a few seconds later than the cars at the front of the train. It is based on the speed of the brake signal, which is usually 10 to $25 \%$ under the speed of sound in open air [18]. Additionally for long trains, because of decrease of the rate of change of air pressure along the main air pipe, the reaction time of the distributor valve for wagons at the rear end of train can be longer than that of the cars at the front of the train. Because even by supposing all mechanical specifications in distributor valves in wagons, the slower drop in the pipe limits the opening of the valve, so the brake cylinder pressure takes longer to build [19]. The late start of the brake cylinder pressure builds up at the rear end of the train and the eventual longer brake application time leads to the rear cars running in-to the front cars and produces large intrain forces. The force can be formulated in such a form as:

$$
F(t)=P(t) f_{\mathrm{b}} A i \eta_{\mathrm{dyn}}
$$

where $F(t)$ and $P(t)$ are time dependent brake force and cylinder pressure respectively. A is effective area of the brake cylinder piston and " $I$ " is rigging ratio whereas $f_{\mathrm{b}}$ and $\eta_{\text {dyn }}$ are friction coefficient and dynamic efficiency of the rigging ratio. These are parameters, which depend on the train brake system. 


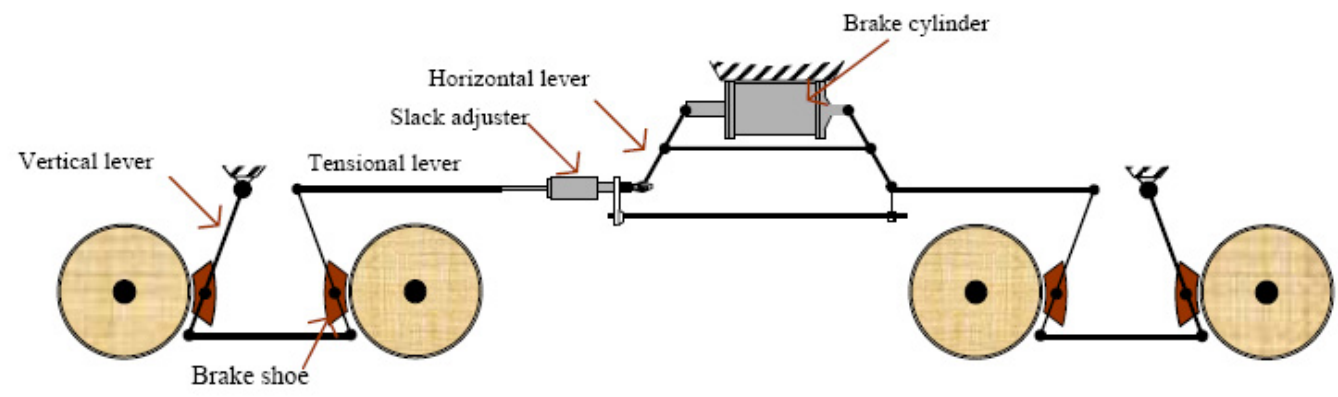

Fig. 3. Schematic of brake system rigging.

The function $P(t)$ is given below.

$$
\left\{\begin{array}{l}
P(t)=\left(\frac{t-t_{\text {delay }}}{t_{\max }}\right)^{\lambda} u\left(t-t_{\text {delay }}\right) p_{\max } \\
P(t)=p_{\max } \quad \text { if } \quad t<t_{\max }+t_{\text {delay }} \\
\text { if } \quad t>t_{\max }+t_{\text {delay }}
\end{array}\right.
$$

Here,

$p_{\max }=$ maximum cylinder pressure;

$t_{\max }=$ time needed for reaching maximum cylinder pressure;

$t_{\mathrm{del}}=$ individual car's application delay time;

$u=$ unit step function;

$\lambda=$ constant reflecting rate of pressure change which depends on the distributor valve;

$t=$ time, starting from the initiation of the train braking by train operator.

Regarding the shoe friction coefficient, the most known formula is as follows [17]:

$$
f_{\mathrm{b}}=0.6 \frac{1+0.01 V}{1+0.05 V} \times \frac{1+0.0056 P_{\mathrm{s}}}{1+0.280 P_{\mathrm{s}}}
$$

where $P_{\mathrm{s}}$ is the pressure $\left[\mathrm{N} . \mathrm{mm}^{-2}\right]$ exerted on the brake shoe and $V\left[\mathrm{~m} \cdot \mathrm{s}^{-1}\right]$ is the linear velocity of the wheel.

\section{Dynamic brake modeling}

Dynamic brake is another kind of brake system, which might be applied in locomotives. Although the basic function of a locomotive is to supply traction effort to the train, the traction motors are also used to slow down the trains if necessary. When the train is moving and a braking effort is needed the rotary motion of each axle can be used to drive the associated traction motor, which is electrically managed to act as a generator. The electric power generated can then be dissipated by means of some electric resistors. The dynamic brake effort, which is used in longitudinal train dynamic modeling of this paper, is shown below in three separate modes as follows [17].

$$
\begin{cases}F_{\mathrm{b}}=\frac{F_{\mathrm{bm}}}{V_{\mathrm{m} 1}} V & V \leq V_{\mathrm{m} 1} \\ F_{\mathrm{b}}=F_{\mathrm{bm}} & V_{\mathrm{m} 1}<V<V_{\mathrm{m} 2} \\ F_{\mathrm{b}}=\frac{F_{\mathrm{bm}} V_{\mathrm{m} 2}}{V} & V \geq V_{\mathrm{m} 2}\end{cases}
$$

Here, $F_{\mathrm{bm}}$ is the maximum braking force and $V_{\mathrm{m} 1}$ and $V_{\mathrm{m} 2}$ the limiting speed where the brake force is constant at its maximum value.

\section{Rolling resistance modeling}

Propulsion resistance of the rail vehicles is usually defined as the sum of rolling and air resistances. These are very much dependent on the shape and design of the vehicles and the complexity of aerodynamic drag force. The calculation of rolling resistance is still dependent on the empirical formulas drawn by manufacturers. In this study, a relation similar to that of Davis equation is used for predicting propulsion resistance. That is:

$F_{\mathrm{r}}=K_{1}\left(2.943+89.2 / m_{\mathrm{pa}}+0.0306 V+1.741 K_{2} V^{2} / m_{\mathrm{t}}\right) m_{\mathrm{t}}$

Here $K_{1}$ and $K_{2}$ are empirical constants, $m_{\text {pa }}$ is mass supported per axle in tons, and $m_{\mathrm{t}}$ represents total mass of the train.

\section{Coupler modeling}

The most important part in longitudinal train dynamic modeling is the wagon connection (coupler) elements. In this research, auto couplers with friction type draft gears are considered. A conventional auto coupler connection and draft gear packages are illustrated in Figure 4.

As can be seen in Figure 4, in modeling a wagon connection, two auto-coupler assemblies must be considered along with gap elements and stiffness elements describing flexure in the wagon body. A wagon connection model will therefore appear as something similar to the schematic shown in Figure 5.

The model of wagon connection can be simplified to a combined draft gear package model equivalent to two draft gear units and one spring element representing locked stiffness, the locked stiffness is the sum of all the stiffness of elements such as the coupler shank, knuckle, yoke, locked draft gear and wagon body all added in series.

Determination of the mathematical model for the draft gear has received considerable attention in technical 


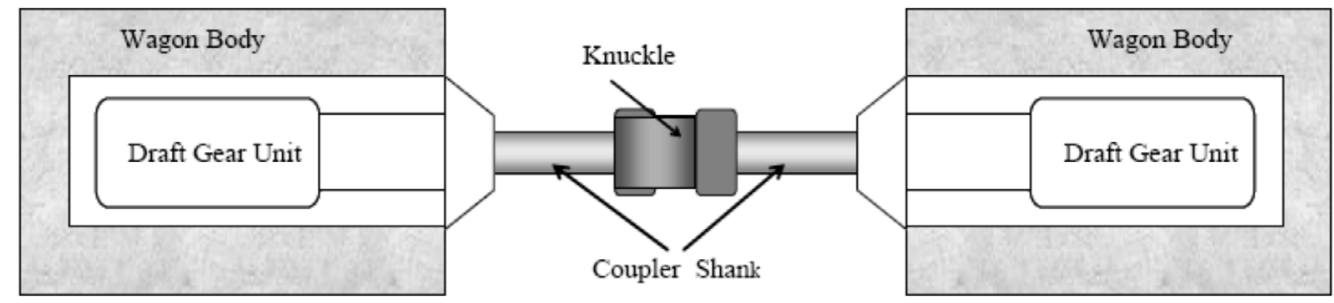

Fig. 4. Conventional auto coupler assembly.

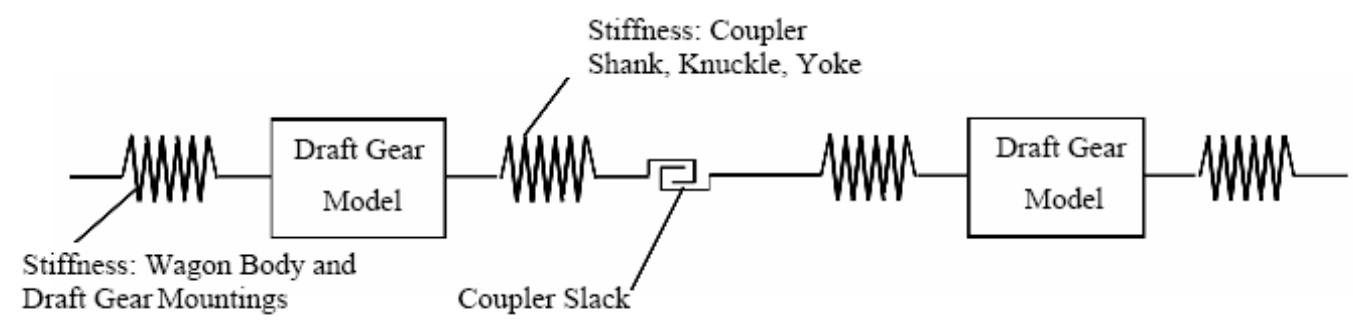

Fig. 5. Components in a wagon connection model.
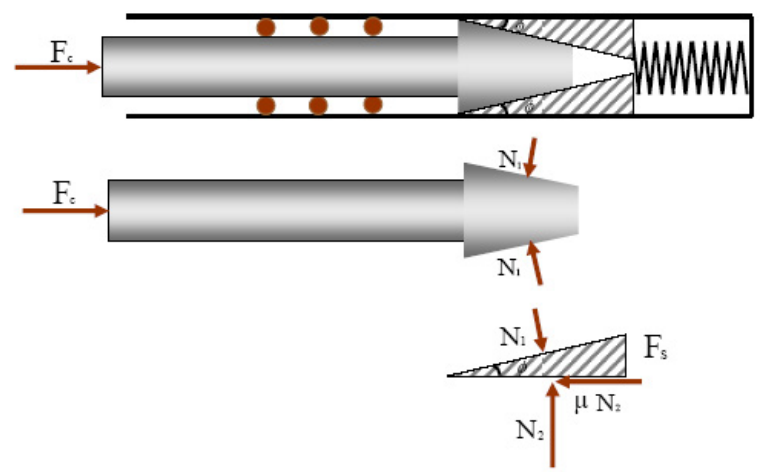

Fig. 6. Free body diagram of a simplified draft gear rod wedge - spring system.

papers [13]. In this research, the draft gear is considered as a single wedge spring as shown in Figure 6 . The rollers provided on one side of the compression rod can be justified in that the multiple wedges are arranged symmetrically around the outside of the rod in the actual unit. It will be realized that different equilibrium states are possible depending on the motion direction of the cars.

In Figure $6, F_{\mathrm{S}}$ and $F_{\mathrm{C}}$ represent, respectively, spring and impact forces between the cars whereas $\mu$ represents friction coefficient. $\mathrm{N}_{1}$ and $\mathrm{N}_{2}$ are vertical components of reaction forces and $\varphi$ is slope of the friction wedge. When there is no impending motion on the sloping surface due to the seating of the rod and wedge, the relation between coupler force and polymer spring force for loading and unloading positions can be developed as follows [13].

$$
F_{\mathrm{C}}=\frac{\tan \varphi}{\tan \varphi \pm \mu} F_{\mathrm{S}}
$$

In this formula, $\tan \varphi$ is considered to be 0.54 and $F_{\mathrm{S}}$ is defined as:

$$
\begin{aligned}
F_{\mathrm{S}}(x)=(0.93 x & +1.162(x-0.02) u(x-0.02) \\
& +0.883 u(x-0.1) u(x-0.1)) 1000
\end{aligned}
$$

Here, $u$ is a unit step function and $x$ represents change in the draft gear position.

The friction coefficient is usually modeled [14] as:

$$
\mu=\left(1+\left(\frac{\mu_{\mathrm{S}}}{\mu_{\mathrm{K}}}-1\right) \exp \left[-2\left|\frac{\mathrm{d} X}{\mathrm{~d} t}\right|\right]\right) \tanh \left(\beta \frac{\mathrm{d} X}{\mathrm{~d} t}\right) \mu_{\mathrm{K}}
$$

where $\mu_{\mathrm{s}}$ and $\mu_{\mathrm{k}}$ are static and dynamic friction coefficients respectively whereas $x$ represents change of draft gear position and $\beta$ represents the friction curve gradient around the point where the friction polarity changes. In the present research $\beta, \mu_{\mathrm{s}}$ and $\mu_{\mathrm{k}}$ are considered $0.20,0.35$ and 0.30 respectively.

As a matter of the fact, too many parameters have important role in the internal draft gear interaction forces:

- the friction-wear interaction,

Draft gears are subject to wear during their life-time and wear alters the dynamical performance of the system [20,21],

- effects of third body at the contact surfaces (contaminants, friction modifiers, lubricants, etc.).

Energy dissipated at the contact surface by frictional forces and the consequent friction recovery due to the cleaning effect caused by the frictional forces destroy the third body layer [22-24].

However, studying all these parameters at the same time could make the problem more complicated, so they 
Table 1. Train specifications.

\begin{tabular}{lc}
\hline Effective mass of locomotive & 108 Tons \\
Effective mass of each wagon & 60.04 Tons \\
Rigging ratio & 11.76 \\
Number of car axles & 0.04 \\
Number of brake shoes for every wagon & 16 \\
Wheel radius & $0.5 \mathrm{~m}$ \\
Brake shoe effective area & $0.009 \mathrm{~mm}^{2}$ \\
Effective area of brake cylinder & $1295 \mathrm{~mm}^{2}$ \\
Length of car body & $21 \mathrm{~m}$ \\
Draft gear travel or displacement & $0.11 \mathrm{~m}$ \\
Coupler slack & $0.02 \mathrm{~m}$ \\
Limiting stiffness or locked stiffness & $170 \mathrm{~N} / \mathrm{m}$ \\
Dynamic braking force in extended region & $300 \mathrm{kN}$ \\
Extended region in dynamic brake characteristics curve & 30 to $60 \mathrm{~km} / \mathrm{h}$ \\
\hline
\end{tabular}

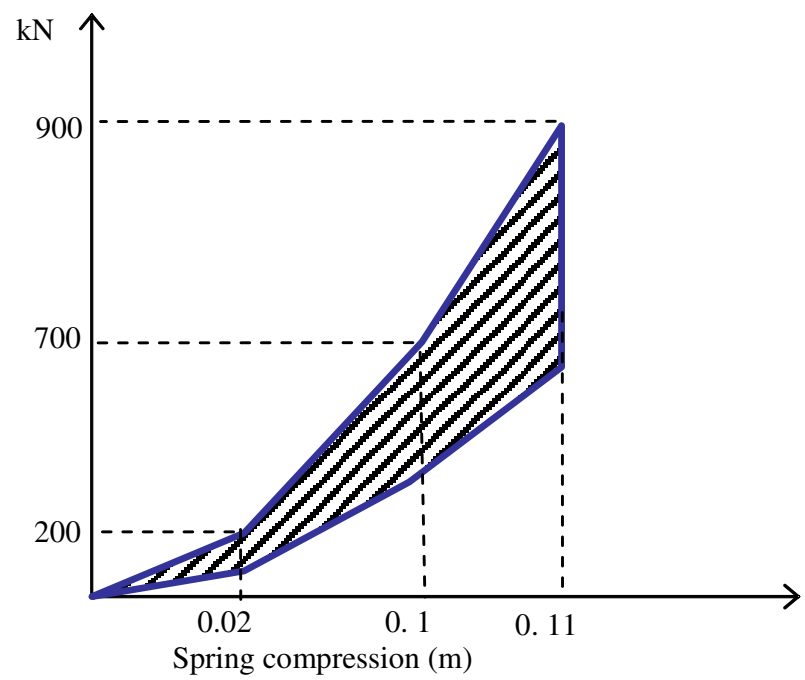

Fig. 7. Characteristics curve of friction type draft gears.

were not considered in the present paper and may be the subject of our future researches.

The dynamic characteristics of draft gear are shown in Figure 7. Here, the enclosed area corresponds to the energy absorbed during longitudinal oscillation processes of the cars.

\section{Simulation conditions}

The train consisted of one locomotive and 52 freight cars. The locomotive and cars were those in use by Iranian Railways. The dynamic brake and traction characteristics of the locomotive and the rolling resistance of the train (locomotive and cars) were discussed in the previous sections above. Other train specifications are given in Table 1.

In this study, the freight cars are equipped with Knnor air brake system and the locomotives are equipped with Sub Wabco air brake system and improved GT26 locomotive's dynamic brake system as in Iranian railways. In the modeling of the train, the characteristics of these systems were used. Here, the maximum air braking effort is
Table 2. Auto coupler stiffness.

\begin{tabular}{lccc}
\hline & $K_{1}(\mathrm{MN} / \mathrm{m})$ & $K_{2}(\mathrm{MN} / \mathrm{m})$ & $K_{3}(\mathrm{MN} / \mathrm{m})$ \\
\hline Simulation 1 & 1.302 & 1.6268 & 1.2362 \\
Simulation 2 & 1.116 & 1.3944 & 1.059 \\
Simulation 3 & 0.93 & 1.162 & 0.883 \\
Simulation 4 & 0.558 & 0.697 & 0.5298 \\
\hline
\end{tabular}

about $206 \mathrm{kN}$ for wagons and the maximum pneumatic and dynamic braking efforts for locomotives are 240 and $300 \mathrm{kN}$ respectively. It is necessary to mention that the maximum braking ratio is usually about 30 to 35 percent of the vehicle weight (locomotives and cars) - multiplied by shoe friction in railways.

Computer simulations are performed by using MAT$\mathrm{LAB}$ version 7.0.0.04. The method used to solve the equations of the train motion is fourth-order variable step size ode23t (Mod. stiff/trapezoidal) with the minimum step size less than $1 \times 10^{-11} \mathrm{~s}$. The initial step size of 0.001 and the max step size in auto mode were selected.

Simulations are conducted for three different cases based on change in coupling stiffness, coupling clearance, and damping parameters, which are characteristic of auto coupler. In this research effect of these parameters on intrain longitudinal forces during brake application is investigated. For each case, three different train forward velocities are also considered.

\subsection{Simulation results for case 1}

In this section, effects of change in coupling stiffness on in-train longitudinal forces are investigated. As can be seen in Table 2, simulations were conducted for four different magnitudes of stiffness.

The simulation results for train forward velocity of $33 \mathrm{~m} . \mathrm{s}^{-1}$ and four different magnitudes of coupling stiffness are presented in Figure 8. Here the delay time which is defined as the time duration that the pressure signal initiated from the locomotive reaches the end of the train is considered to be $5.72 \mathrm{~s}$.

In Figure 8, it shows that during the cylinder pressure build up time, the braking effort is low. It is interesting 

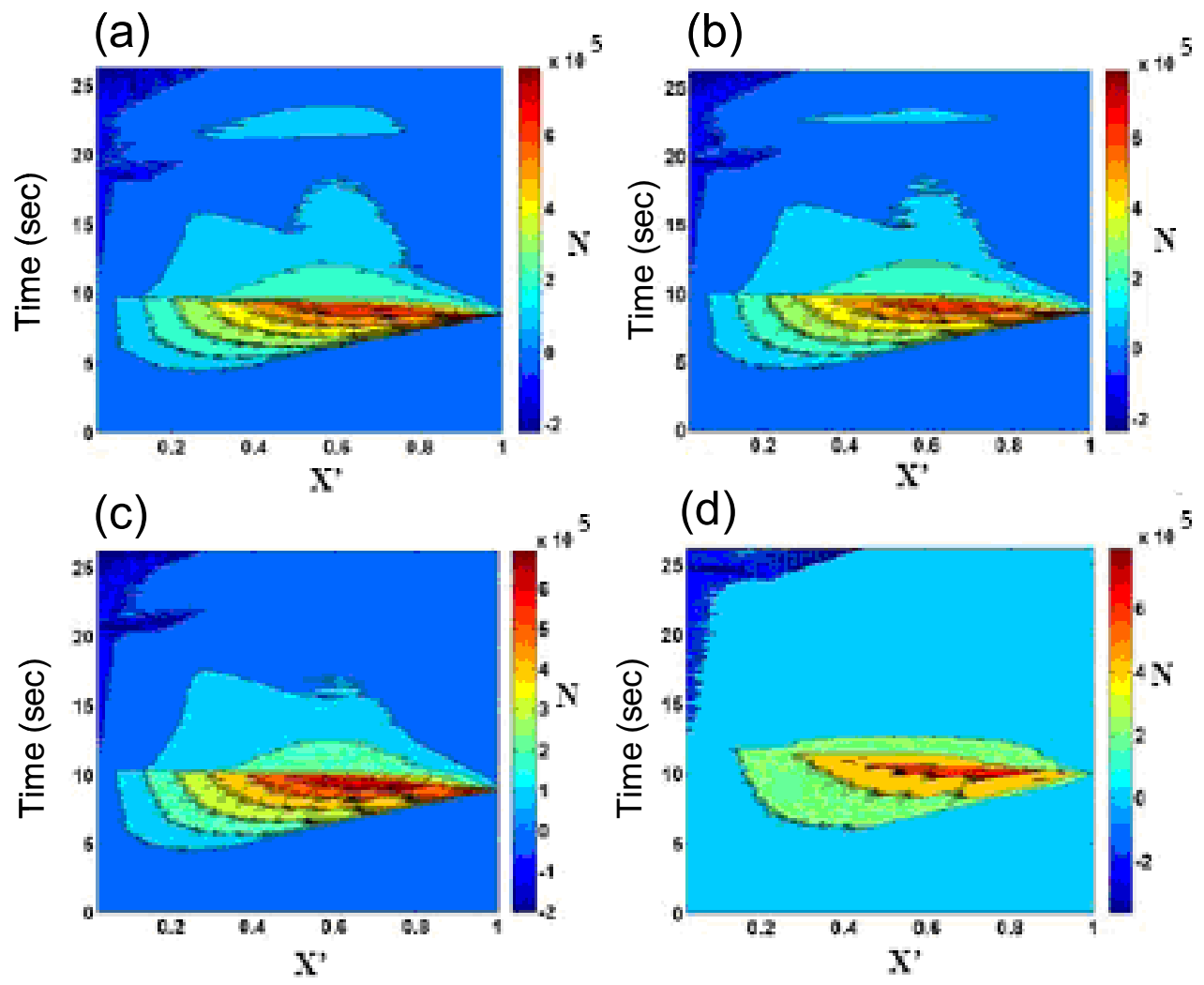

Fig. 8. In-train coupler forces counter plots (a) simulation 1, (b) simulation 2, (c) simulation 3 and (d) simulation 4 (train forward velocity: $\left.33 \mathrm{~m} \cdot \mathrm{s}^{-1}\right)$. $X^{\prime}$ : Normalized distance of the cars from the train head.

to see that the peaks of maximum longitudinal compression force occur at the end cars and at about mid stopping time. Comparison between results of simulations 1 , 2 , and 3 shows that with decrease of stiffness, in train compression forces decrease and tensile forces increase. However, comparison between results of simulations 3 and 4 shows that with decrease of stiffness, in train compression forces increase and tensile forces dangerously increase. It is due to that in simulation 4 in some instance draft-gears reach end of displacement, therefore stiffness dangerously increased.

For different magnitudes of coupling stiffness parameter corresponding to simulations $1,2,3$ and 4 the maximum in-train compression forces for the individual cars along the train length are presented in Figure 9a. Here, the vertical axis represents force in Newton and horizontal axis represents the non-dimensional wagon location in the train. As can be seen in this Figure, the locations of the maximum compression force are to be at the last third part of the train length at the end of the train. The maximum values of these compression forces are calculated to be $813,813,771$, and $868 \mathrm{kN}$ for different magnitudes of coupling stiffness parameter corresponding to simulations 1, 2, 3 and 4 respectively. The calculated RMS (root mean square) in-train compression forces corresponding to simulations 1, 2, 3 and 4 along the train for individual cars are plotted in Figure 9b.
Here, the RMS (root mean square) in train compression forces are calculated by use of formula (12) below.

RMS in-train compression forces $=\sqrt[2]{\frac{\int_{0}^{t_{b}}(|f(t)|+f(t))^{2} \mathrm{~d} t}{2 t_{\mathrm{b}}}}$

In the above formula $f(t)$ and $t_{\mathrm{b}}$ are auto coupler force and brake application time.

From Figure 9b, it can be seen that the maximum RMS of compression forces occurs at about 0.6 of the train length indicating that the location of the maximum coupler force is nearly fixed in the whole braking period. The maximum values of the RMS compression forces are calculated to be $211,211.6$ and $219.3 \mathrm{kN}$ corresponding to simulations $1,2,3$ and 4 , respectively.

The simulation results for the maximum and RMS intrain tensile forces along the train length are presented in Figures 10a and 10b, respectively.

Here, RMS (root mean square) in-train compression forces are calculated by use of formula 13 below.

$$
\text { RMS in-train tensile forces }=\sqrt[2]{\frac{\int_{0}^{t_{b}}(|f(t)|-f(t))^{2} \mathrm{~d} t}{2 t_{\mathrm{b}}}}
$$



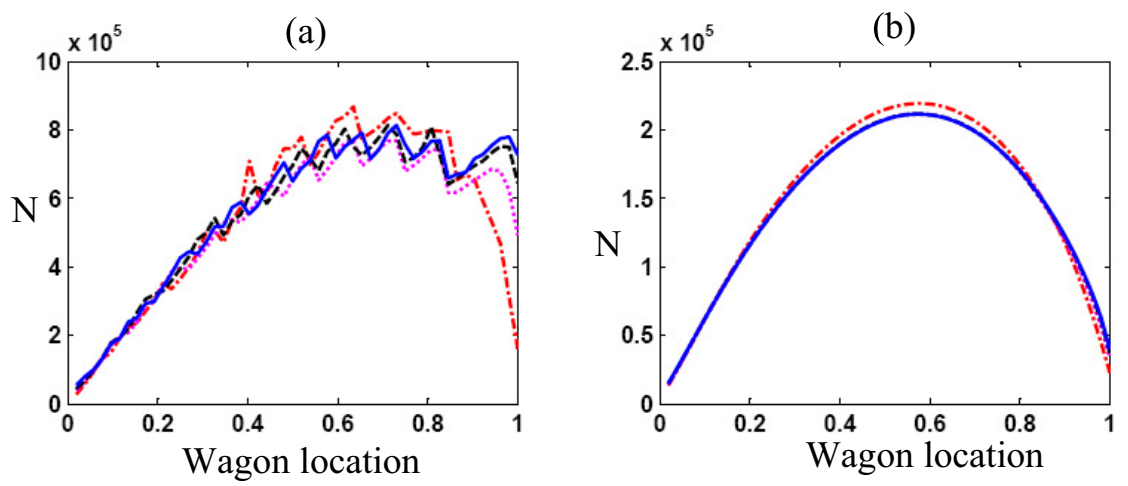

Fig. 9. (a) Maximum longitudinal compression coupler forces, (b) RMS of longitudinal compression coupler forces corresponding to simulation $-1,--2, \ldots 3$ and - . 4 , respectively.
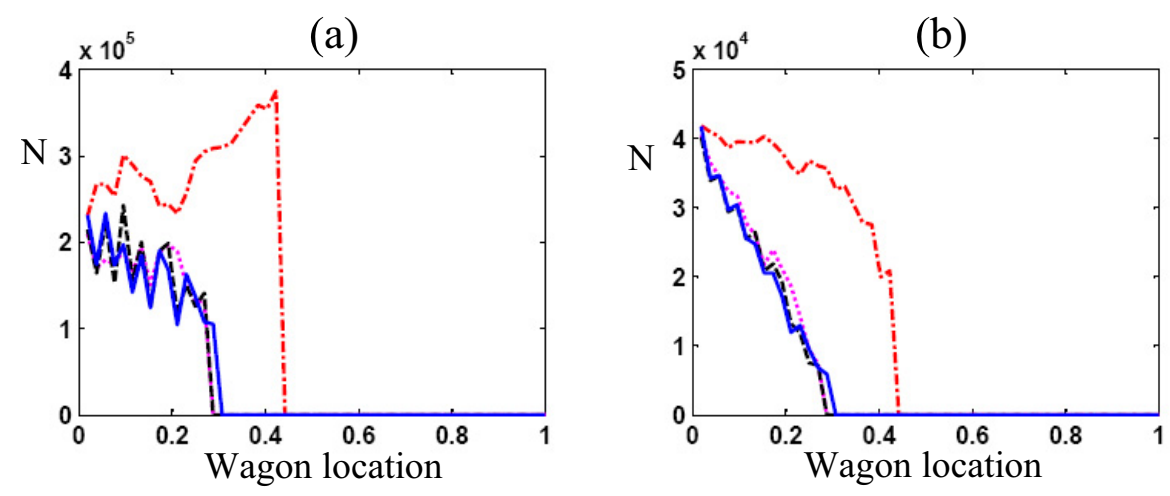

Fig. 10. (a) Maximum longitudinal tensile coupler forces, (b) RMS of longitudinal tensile coupler forces corresponding to simulation $-1,--2, \ldots 3$ and - . 4 , respectively.

In the above formula $f(t)$ and $t_{\mathrm{b}}$ are auto coupler force and brake application time.

It can be seen from Figure 10a that, with increase coupling stiffness tensile forces and length of train, which experience tensile forces increase. The maximum values of these tensile forces are calculated to be 233, 243, 209 and $374 \mathrm{kN}$ corresponding to simulations $1,2,3$ and 4 , respectively.

In Figure 10b it can be seen that the RMS in-train tensile force increases similary as tensile force with increase of magnitude of coupling stiffness parameter. The maximum values of these RMS tensile forces are calculated to be $42.1,40.33,40.53$ and $42.18 \mathrm{kN}$ for different magnitudes of coupling stiffness parameter, respectively.

This simulation was repeated for two other initial train forward velocities of 25 and $17 \mathrm{~m} . \mathrm{s}^{-1}$. In these simulations, the same behaviors as the previous simulation are seen. Therefore, for brevity, data in Tables 3 and 4 for maximum magnitudes of in-train compression and tensile forces are presented.

As it can be seen in Table 3, with decrease of coupling stiffness parameter, magnitude of in-train compression force increases, however if draft gear reaches end of travel compression forces dangerously increase. In addition, it is noticeable to see that with increase of train forward velocity, magnitude of in- train compression forces decreases.

As it can be seen in Table 4 with the decrease of the coupling stiffness parameter, the magnitude of in-train tensile force a little changes, however if draft gear reaches end of travel tensile forces dangerously increase.

\subsection{Simulation results for case 2}

In this section, the effect of the damping parameter (the area bordered by coupling cycle) change on intrain longitudinal forces is investigated. For the damping change of the draft gear two different parameters, slope of gradient surface and friction coefficient can be used. Effect of increase in slops of the auto coupler on damping has similar effect to that of friction coefficient decrease and vice versa. In this research a change on friction coefficient was considered and simulation conducted for three different magnitudes of $\mu_{\mathrm{s}}: 0.29,0.35$ and 0.41 , respectively.

The simulation results for train forward velocity of $33 \mathrm{~m} . \mathrm{s}^{-1}$ and three different magnitudes of damping parameter is presented in Figure 11. Brake delay time is considered the same as for the before case. It is interesting to see from Figure 11 that the peaks of maximum 
Table 3. Magnitudes of maximum compression forces for different simulations.

\begin{tabular}{cccccccccccccc}
\hline Velocity [km/h] & \multicolumn{1}{c}{120} & \multicolumn{1}{c}{90} & \multicolumn{1}{c}{60} \\
\hline Simulation nom & 1 & 2 & 3 & 4 & 1 & 2 & 3 & 4 & 1 & 2 & 3 & 4 \\
\hline $\begin{array}{c}\text { Maximum in-train } \\
\text { compression force }[\mathrm{kN}]\end{array}$ & 813 & 813 & 771 & 868 & 900 & 886 & 879 & 980 & 1014 & 1032 & 1030 & 1170 \\
\hline
\end{tabular}

Table 4. Magnitudes of maximum tensile forces for different simulations.

\begin{tabular}{cccccccccccccc}
\hline Velocity $[\mathrm{km} / \mathrm{h}]$ & \multicolumn{1}{c}{120} & \multicolumn{1}{c}{90} & \multicolumn{1}{c}{60} \\
\hline Simulation nom & 1 & 2 & 3 & 4 & 1 & 2 & 3 & 4 & 1 & 2 & 3 & 4 \\
\hline $\begin{array}{c}\text { Maximum in-train } \\
\text { tensile force }[\mathrm{kN}]\end{array}$ & 233.5 & 243 & 209 & 374 & 209 & 195 & 234 & 214 & 143 & 123 & 149 & 415 \\
\hline
\end{tabular}

(a)

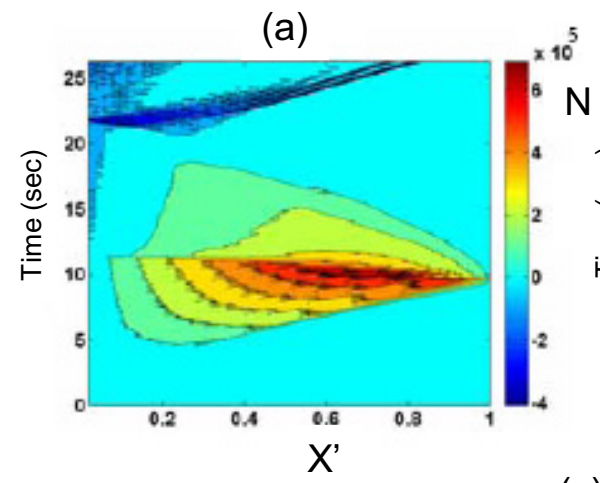

(b)

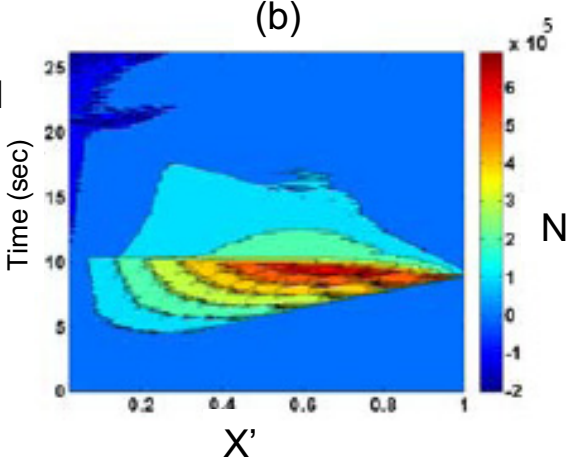

(c)

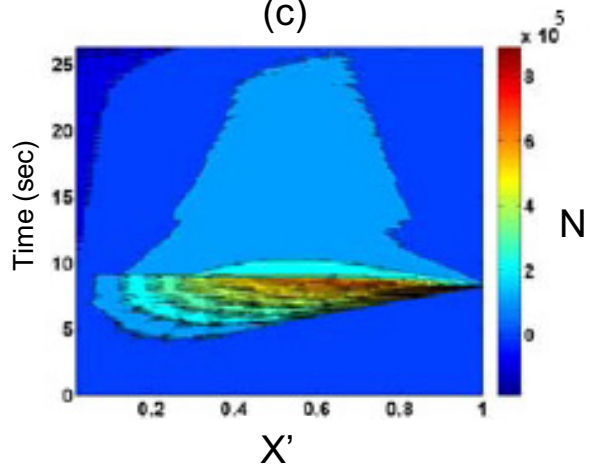

Fig. 11. In-train coupler forces counter plots (a), (b) and (c), corresponding to three different magnitudes of $\mu_{\mathrm{s}}: 0.29,0.35$ and 0.41 , respectively (train forward velocity of $33 \mathrm{~m} . \mathrm{s}^{-1}$ ). $X^{\prime}$ : Normalized distance of the cars from the train head.

longitudinal compression force occur at the end cars and at about mid stopping time. It is similar to the previous case.

In train forces along the train length are presented in Figure 12a. Here, the vertical axis represents force in Newton and horizontal axis represents the non-dimensional wagon location in the train. As can be seen in these Figures, similarly to the previous case, locations of the maximum compression force are to be at the last third part of the train length at the end of the train for three different damping magnitudes. The maximum values of these compression forces are calculated to be 786,772 and $950 \mathrm{kN}$ for different magnitudes of $\mu_{\mathrm{s}}$ : $0.29,0.35$ and 0.41 , respectively. Increasing friction coefficient leads to damping growth (the area bordered coupling cycle) and compression stiffness (draft gear stiffness in positive displacement) increase while being pressed. According to the investigation done, it was clear that by increasing friction coefficient from 0.29 to 0.35 , damping growth effect overwhelms its stiffness growth, and the amount of compression forces among wagons decreases, but while increasing the friction coefficient from 0.35 to 0.41 the influence of coupling stiffness increase overcomes damping growth. Hence, the amount of compression forces goes up mention worthy.

The calculated RMS (root mean square) in-train compression forces along the train for individual cars are plotted in Figure $12 \mathrm{~b}$ under three different magnitudes of damping parameter. It can be seen that the maximum magnitude of RMS tensile force occurs at about 0.6 of the train length indicating the location of the maximum RMS of compression coupler force nearly fixed for different magnitudes of damping parameter. The maximum values of the RMS compression forces are calculated to be 216.9, 

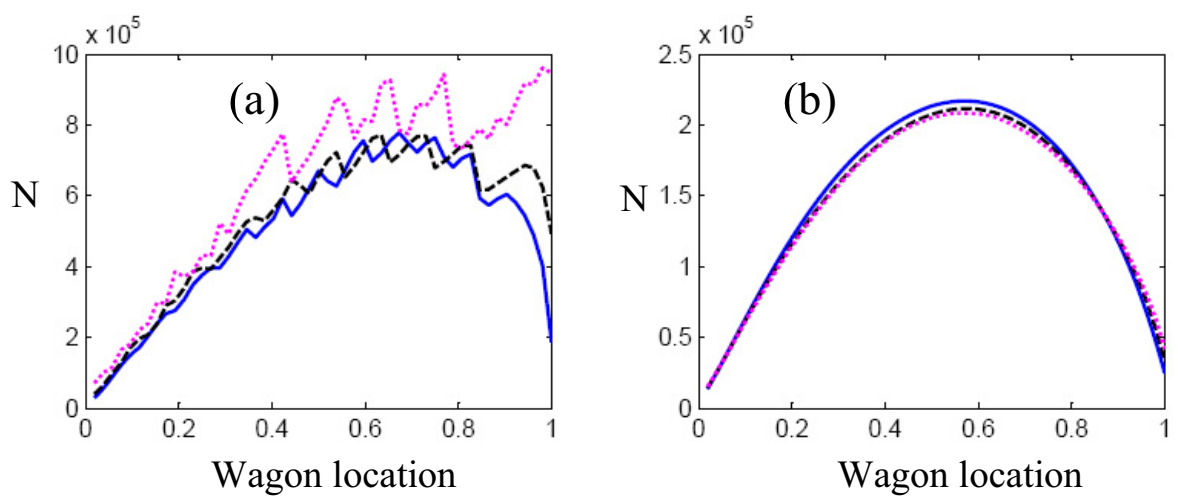

Fig. 12. (a) Maximum longitudinal compression coupler forces, (b) RMS of longitudinal compression coupler forces, for three different magnitudes of $\mu_{\mathrm{s}}-0.29--0.35$ and $\ldots .0 .41$, respectively.
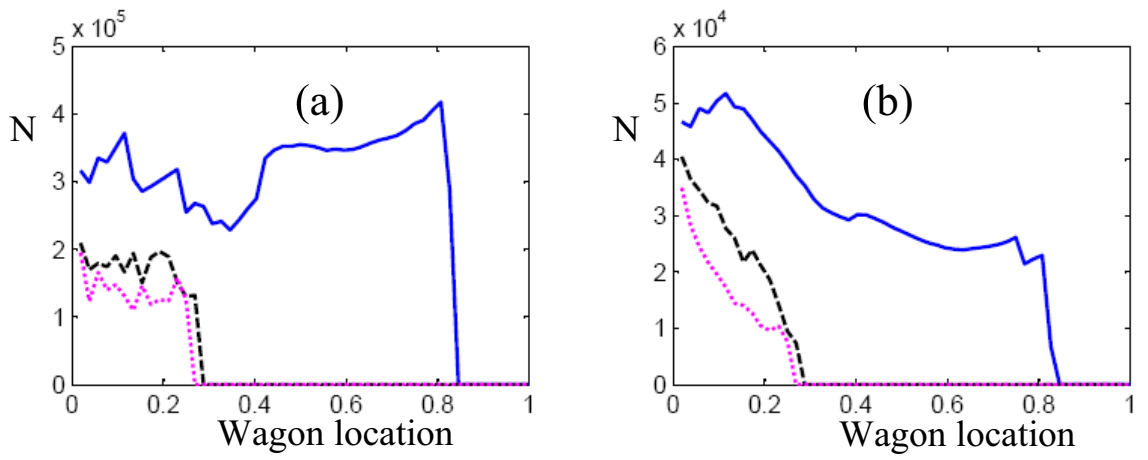

Fig. 13. (a) Maximum longitudinal tensile coupler forces, (b) RMS of longitudinal tensile coupler forces, for three different magnitudes of $\mu_{\mathrm{s}}-0.29--0.35$ and $\ldots .0 .41$, respectively.

211.6 and $208.5 \mathrm{kN}$ for different magnitude of $\mu_{\mathrm{s}}: 0.29$, 0.35 and 0.41 , respectively.

The simulation results for the maximum and RMS in-train tensile forces along the train are presented in Figures $13 \mathrm{a}$ and $13 \mathrm{~b}$ respectively. It can be seen from Figure $12 \mathrm{a}$ that, with increases in magnitude of damping parameter magnitude of tensile forces and length of train experience tensile force a little decreased. The maximum values of these tensile forces are calculated to be 418, 209.9 and $195.3 \mathrm{kN}$ for three different magnitudes of $\mu_{\mathrm{s}}: 0.29,0.35$ and 0.41 , respectively. In Figure $13 \mathrm{~b}$ can be seen that the RMS in-train tensile force almost have not been affected by increase magnitude of damping parameter. The maximum values of these RMS tensile forces are calculated to be $51.7,40.53$ and $35.03 \mathrm{kN}$ for three different magnitudes of $\mu_{\mathrm{s}}: 0.29,0.35$ and 0.41 , respectively.

This simulation is repeated for two other initial train forward velocities of 25 and $17 \mathrm{~m} . \mathrm{s}^{-1}$. In these simulations, the same behaviors as the previous simulation are seen. Therefore, for brevity, data in Tables 5 and 6 maximum for magnitudes of in-train compression and tensile forces are presented.

As stated in Table 5, for different train forward velocities while friction increases from 0.29 to 0.35 in-train compression forces decrease but with increase in friction from 0.35 to 0.41 magnitudes of in-train compression forces go up.
It is also noticeable that with increase of train forward velocity, magnitude of in- train compression forces decreases.

In Table 6 magnitudes of in-train, tensile forces are presented. As it can be seen with decrease of friction coefficient, magnitude of in-train tensile forces increases.

\subsection{Simulation results for case 3}

In this section, effects of coupling clearance on in-train longitudinal forces are investigated. The simulation results for train forward velocity of $33 \mathrm{~m} . \mathrm{s}^{-1}$ and three different magnitudes of coupling clearance such as 0.08, 0.04, and $0.02 \mathrm{~m}$ are presented in Figure 14. Brake delay time is considered the same as for the previous cases. In this Figure it can be seen that with increase coupling clearance magnitude, in-train compression and tensile forces a little increase.

The maximum in-train compression forces for three different magnitudes of coupling clearance for the individual cars along the train length are presented in Figure $15 \mathrm{a}$. Here, the vertical axis represents force in Newton and horizontal axis represents the non-dimensional wagon location in the train. As it can be seen in these Figures, similar to the previous cases, locations of the maximum compression force are to be at the last third part of the 
S. Mohammadi and R. Serajian: Mechanics \& Industry 16, 205 (2015)

Table 5. Magnitudes of maximum compression forces for different simulations.

\begin{tabular}{cccccccccc}
\hline Velocity [km/h] & & 120 & & 90 & & \multicolumn{3}{c}{60} & \\
\hline Friction coefficient & 0.29 & 0.35 & 0.4 & 0.29 & 0.34 & 0.4 & 0.29 & 0.34 & 0.4 \\
\hline $\begin{array}{c}\text { Maximum in-train } \\
\text { compression force }[\mathrm{kN}]\end{array}$ & 778 & 771 & 961 & 910 & 879 & 1035 & 1091 & 1030 & 1131 \\
\hline
\end{tabular}

Table 6. Magnitudes of maximum tensile forces for different simulations.

\begin{tabular}{cccccccccc}
\hline Velocity [km/h] & & 120 & \multicolumn{1}{c}{90} & \multicolumn{3}{c}{60} \\
\hline Friction coefficient & 0.29 & 0.35 & 0.4 & 0.29 & 0.34 & 0.4 & 0.29 & 0.34 & 0.4 \\
\hline $\begin{array}{c}\text { Maximum in-train } \\
\text { tensile force }[\mathrm{kN}]\end{array}$ & 418 & 209 & 195 & 390 & 234 & 133 & 287 & 149 & 129 \\
\hline
\end{tabular}

(a)

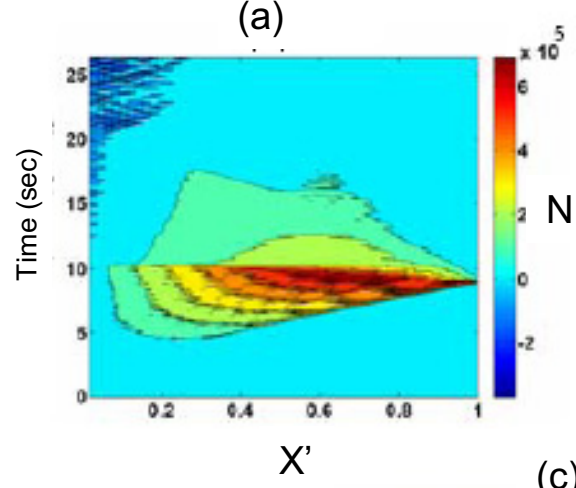

(c) (b)

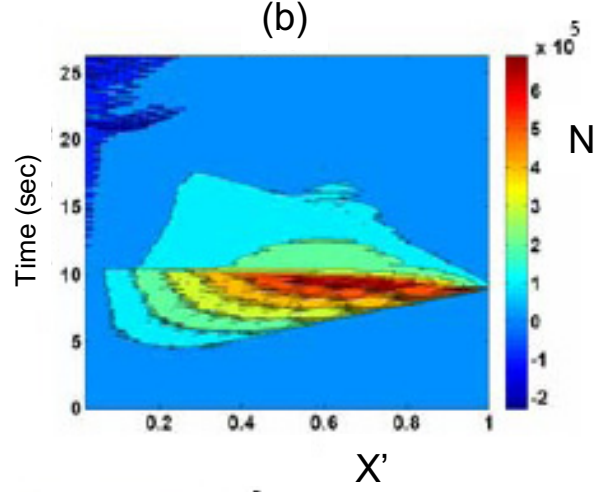

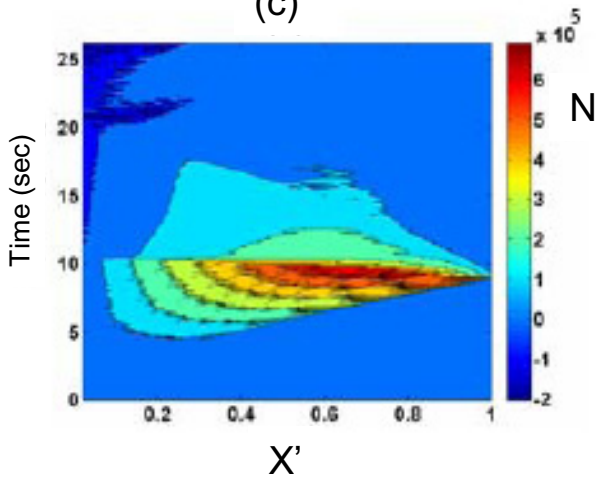

Fig. 14. In-train coupler forces counter plots (a), (b) and (c), corresponding to three different magnitudes of coupling clearance parameter magnitudes of $0.08,0.04$ and $0.02 \mathrm{~cm}$, respectively (train forward velocity of $33 \mathrm{~m} . \mathrm{s}^{-1}$ ). $X^{\prime}$ : Normalized distance of the cars from the train head.

train length at the end of the train for three different coupling clearance magnitudes. The maximum values of these compression forces are calculated to be 798,785 , and $771 \mathrm{kN}$ for different magnitudes of coupling clearance parameter as $0.08,0.04$, and $0.02 \mathrm{~m}$ respectively. The calculated RMS (root mean square) in train compression forces along the train for individual cars are plotted in Figure 15b, indicating that the location of the maximum RMS of compression coupler force is nearly fixed for different magnitudes of coupling clearance parameter. The maximum values of the RMS compression forces are calculated to be $211.11,211.10$, and $211.12 \mathrm{kN}$ for different magnitudes of coupling clearance $0.08,0.04$, and $0.02 \mathrm{~cm}$ respectively.

The simulation results for the maximum and RMS in-train tensile forces along the train are presented in
Figures $16 \mathrm{a}$ and $16 \mathrm{~b}$ respectively. It can be seen from Figure 16a that, with increases in magnitude of coupling clearance parameter magnitude of tensile forces and length of train experience tensile force increase. The maximum values of these tensile forces are calculated to be $379.6,235.9$ and $209.9 \mathrm{kN}$ for different magnitudes of 0.08 , 0.04 , and $0.02 \mathrm{~cm}$, respectively for coupling clearance parameter, respectively.

In Figure 16b, it can be seen that the RMS in-train tensile force almost has been affected by increase magnitude of coupling clearance parameter and increased. The maximum values of these RMS tensile forces are calculated to be $52.9,40.53$ and $40.53 \mathrm{kN}$ for three different magnitudes of coupling clearance parameter, respectively.

This simulation is repeated for two other initial train forward velocities of 25 and $17 \mathrm{~m} . \mathrm{s}^{-1}$. In these 

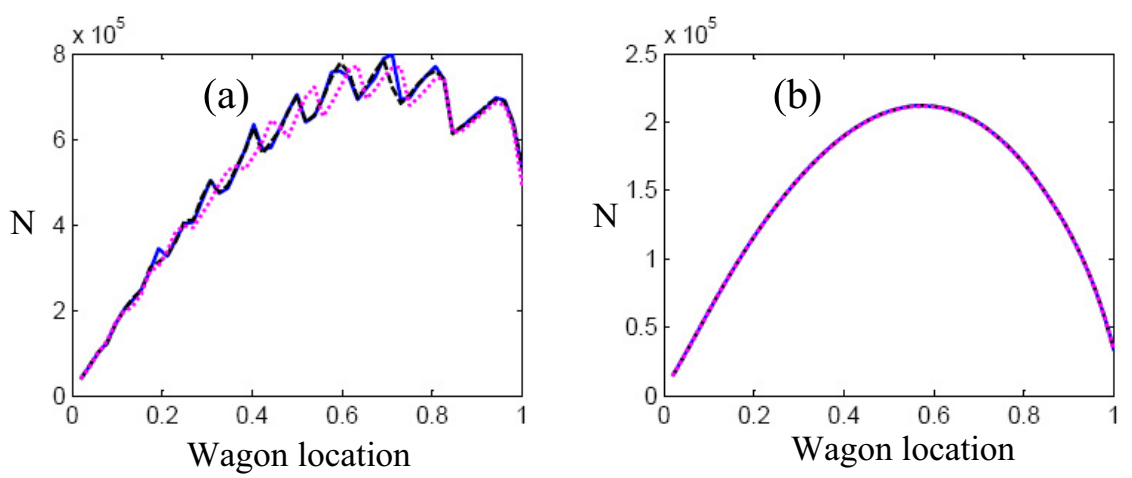

Fig. 15. (a) Maximum longitudinal compression coupler forces, (b) RMS of longitudinal compression coupler forces (for coupling clearance parameter magnitude $-0.08--0.04$ and $\ldots 0.02 \mathrm{~cm})$.
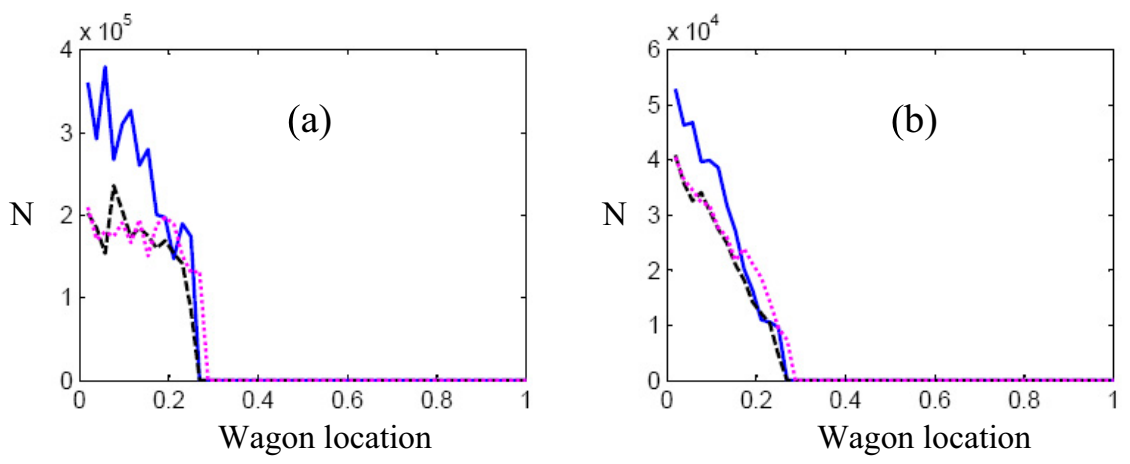

Fig. 16. (a) Maximum longitudinal tensile coupler forces, (b) RMS of longitudinal tensile coupler forces (for coupling clearance parameter magnitude $-0.08 \mathrm{~cm},--0.04 \mathrm{~cm}$ and $\ldots 0.02 \mathrm{~cm})$.

Table 7. Magnitudes of maximum compression forces for different simulations.

\begin{tabular}{cccccccccc}
\hline Velocity [km/h] & & 120 & & 90 & \multicolumn{3}{c}{60} \\
\hline Coupling clearance $[\mathrm{m}]$ & 0.08 & 0.04 & 0.02 & 0.08 & 0.04 & 0.02 & 0.08 & 0.04 & 0.02 \\
\hline $\begin{array}{c}\text { Maximum in-train } \\
\text { compression force }[\mathrm{kN}]\end{array}$ & 798 & 785 & 771 & 898 & 888 & 879 & 1041 & 1042 & 1030 \\
\hline
\end{tabular}

Table 8. Magnitudes of maximum tensile forces for different simulations.

\begin{tabular}{cccccccccc}
\hline Velocity $[\mathrm{km} / \mathrm{h}]$ & & 120 & \multicolumn{3}{c}{90} & \multicolumn{3}{c}{60} \\
\hline Coupling clearance [m] & 0.08 & 0.04 & 0.02 & 0.08 & 0.04 & 0.02 & 0.08 & 0.04 & 0.02 \\
\hline $\begin{array}{c}\text { Maximum in-train } \\
\text { tensile force }[\mathrm{kN}]\end{array}$ & 379 & 235 & 209 & 334 & 271 & 234 & 180 & 158 & 149 \\
\hline
\end{tabular}

simulations, the same behaviors as the previous simulation are seen. Therefore, for brevity, data in Tables 7 and 8 for maximum magnitudes of in-train compression and tensile forces are presented.

As it can be seen in Table 8 with increase of coupling clearance, magnitude of in-train compression force a little increases. In addition similarly as for the two previous cases with increase of train forward velocity, magnitude of in-train compression forces decreases.

In Table 8 magnitudes of in-train tensile forces are presented. As it can be seen with decreases of coupling clearance, magnitude of in-train tensile force dangerously increases.

\section{Future works}

In this research, effect of the change in auto coupler parameters on in-train longitudinal forces was investigated computationally. For our future works, we want to measure in-train longitudinal forces experimentally (field research). For this reason, we are going to have some cooperation with Iranian railway. In the future study, local conditions for over one hundred active wagons in Iran railway system in specific durations would be investigated. On related wagons and near the coupling, we would attach suitable ultrasonic equipement or light sensor for getting the information about the amount of couplings' compression or tensile distance while train is moving and calculate 
the generated longitudinal force according to draft gear specifications.

\section{Conclusion}

In this research effect of change in coupling key parameters such as stiffness, damping and clearance, on in-train longitudinal forces during brake application is computationally studied precisely.

The train contained one locomotive and fifty-two wagons equipped with automatic couplers running on a straight track at three different forward velocities. The train was modeled as lumped mass. The locomotive dynamic brake and air brake of the cars were theoretically modeled in the simulation.

Results of our study show that while reducing the coupling stiffness, maximum compression force between wagons would decrease, but if the gears draft reaches its end travel, maximum compression force increases intensively. On the other hand, with this phenomenon, coupling loses its capability in damping lashes between wagons and leads to severe tensile forces too. Therefore, choosing optimum stiffness and coupling travel is very essential. Investigation done on this matter shows that the location of maximum in-wagon tensile force creation is not related to coupling stiffness and always happens in the last one third $(1 / 3)$ while draft gears have not reached their end travel.

Increasing friction coefficient leads to damping growth (the area between coupling cycle) and compression stiffness increase while being pressed. According to the investigation done, it was clear that by increasing friction coefficient from 0.29 to 0.35 , damping growth effect overwhelms its stiffness growth, and the amount of compression forces among wagons decreases, but while increasing the friction coefficient from 0.35 to 0.41 the influence of coupling stiffness increase overcomes damping growth. Hence, the amount of compression forces goes up mention worthy. It was seen that by increasing damping, the amount of tensile and longitudinal forces in-train decreases greatly.

With increase coupling clearance magnitude, in-train compression a little increases. In addition, it is noticeable that with increase of coupling clearance magnitude of tensile force and length of train, which experience tensile forces increases. Locations of the maximum compression force are to be at the last third part of the train length at the end of the train for three different coupling clearance magnitudes.

\section{References}

[1] J.S. Rao, E. Raghavacharyulu, N, Kumar, Mathematical modeling to simulate the transient dynamic longitudinal force draw cms of a train-consist, J. Sound Vib. 3 (1980) 365-379

[2] I.B. Duncan, P.A. Webb, The Longitudinal Behavior of Heavy Haul Trains Using Remote Locomotives, Fourth International Heavy Haul Conference, Brisbane, 1989, pp. $587-590$
[3] B.J. Jolly, B.G. Sismey, Doubling the Length of Coals Trains in the Hunter Valley, Fourth International Heavy Haul Conference, Brisbane, 1989, pp. 579-583

[4] K. Fukazawa, Coupler forces of 1000t class two-axle freight trains, Quarterly Report of RTRI 33 (1992) 166168

[5] M. El-Sibaie, Recent Advancements in Buff and Draft Testing Techniques, Fifth International Heavy Haul Conference, Beijing, 1993

[6] Zobory, Istvan, Bekefi, Elemer, On real-time simulation of the longitudinal dynamics of trains on a specified railway line, Period. Polytech. Transp. Eng. 23 (1995) 3-18

[7] S. Barbosa, I. Roberto, Weber, Hans, Longitudinal train dynamics, J. Brazilian Soc. Mecha. Sci. 18 (1996) 107-116

[8] C. Oyan, Dynamic simulation of Taipei EMU train, Vehicle Sys. Dyn. 30 (1998) 10.043-167

[9] V.G. Verbitskiy, L.G. Lobas, Simulation of dynamic behavior of monorail car, Eng. Simul. 18 (2000) 119-130

[10] M. McClanachan, C. Cole, D. Roach, B. Scown, An Investigation of the Effect of Bogie and Wagon Pitch Associated with Longitudinal Train Dynamics, The Dynamics of Vehicles on Roads and on Tracks-Vehicle System Dynamics Supplement 33, Swets \& Zeitlinger, Amsterdam, 1999, pp. 370.04-385

[11] M. Ansari, D. Younesian, E. Esmailzadeh, Sensitivity Analysis in Longitudinal Freight Trains Dynamics, ASME conference, 2008

[12] M. Ansari, D. Younesian, E. Esmailzadeh, Effects of load distribution patterns on the Longitudinal Freight Trains Dynamics, CSME conference, 2008

[13] H.I. Andrews, Railway Traction, Elsevier Science Publishers, 1986

[14] UIC Leaflet 50.040-1, Air-Braking system, 2004

[15] Saumber and Eduerd Gerum and Peter J. Berndt, Fundamentals of Railway Vehicle Braking, 1993

[16] S. Iwnicki, Handbook of Railway Vehicle Dynamics, Published by CRC Press, ISBN 080.049333210, 978080.049333217, 2006

[17] J. Ramon, Nonlinear Investigation of Two Doff Stick Slip System, Elsevier Science Publishers, 2009

[18] P.J. Blau, Embedding wear models into friction models, Tribol. Lett. 34 (2009) 75-79

[19] M. Ignesti, M. Malvezzi, L. Marini, E. Meli, A. Rindi, Development of a wear model for the prediction of wheel and rail profile evolution in railway systems, Wear 284285 (2012) 1-17

[20] O. Polach, Creep forces in simulations of traction vehicles running on adhesion limit, Wear 258 (2005) 992-1000

[21] E. Meli, A. Ridolfi, A. Rindi, An innovative degraded adhesion model for railway vehicles: development and experimental validation, Meccanica 49 919-937

[22] B. Allotta, E. Meli, A. Ridolfi, A. Rindi, Development of an innovative wheel-rail contact model for the analysis of degraded adhesion in railway systems, Tribol. Int. 69 (2013) 128-140

[23] Railway Technical Web Pages, Air Brakes, This Page introduced 3 May 1999 and last updated 1 February 2001

[24] UIC Leaflet 504-1, Brakes- Braking Power, 2004 